ESAIM: PROCEEDINGS, December 2012, Vol. 38, p. 99-117

F. Coquel, M. Gutnic, P. Helluy, F. Lagoutière, C. Rohde, N. Seguin, Editors

\title{
NUMERICAL SIMULATION OF THE SELECTION PROCESS OF THE OVARIAN FOLLICLES
}

\author{
Benjamin Aymard ${ }^{1,2}$, Frédérique Clément ${ }^{2}$, Frédéric Coquel ${ }^{3}$ and Marie \\ POSTEL $^{1}$
}

\begin{abstract}
This paper presents the design and implementation of a numerical method to simulate a multiscale model describing the selection process in ovarian follicles. The PDE model consists in a quasi-linear hyperbolic system of large size, namely $N_{f} \times N_{f}$, ruling the time evolution of the cell density functions of $N_{f}$ follicles (in practice $N_{f}$ is of the order of a few to twenty). These equations are weakly coupled through the sum of the first order moments of the density functions. The timedependent equations make use of two structuring variables, age and maturity, which play the roles of space variables. The problem is naturally set over a compact domain of $\mathbf{R}^{2}$. The formulation of the time-dependent controlled transport coefficients accounts for available biological knowledge on follicular cell kinetics. We introduce a dedicated numerical scheme that is amenable to parallelization, by taking advantage of the weak coupling. Numerical illustrations assess th e relevance of the proposed method both in term of accuracy and HPC achievements.

Résumé. Ce document présente la conception et l'implémentation d'une méthode numérique servant à simuler un modèle multiéchelle décrivant le processus de sélection des follicules ovariens. Le modèle EDP consiste en un système hyperbolique quasi linéaire de grande taille, typiquement $N_{f} \times N_{f}$, gouvernant l'évolution des fonctions de densité cellulaire pour $N_{f}$ follicules (en pratique $N_{f}$ est de l'ordre de quelques-uns à une vingtaine). Ces équations d'évolution utilisent deux variables structurantes, l'âge et la maturité, qui jouent le rôle de variables d'espace. Le problème est naturellement posé sur un domaine compact de $\mathbf{R}^{2}$. La formulation du transport à coefficients variables au cours du temps en fonction du contrôle est issue des connaissances disponibles sur la cinétique cellulaire au sein des follicules ovariens. Nous présentons un schéma numérique dédié au problème parallélisable en tirant parti du faible couplage. Des simulations numériques permettent d'évaluer la pertinence de la méthode proposée tant en terme de précision que de calcul haute performance.
\end{abstract}

\section{INTRODUCTION}

This work is motivated by a mathematical modelling approach of a complex physiological system, the development of ovarian follicles. The model describes both the cell dynamics within each follicle and the competition process within the population of follicles. The resulting model $(\overline{9}])$ is a large scale system of weakly coupled quasi-linear transport equations, where integro-differential terms occur both in the velocity and source term. The coupling terms account for the endocrine-based dependence of one follicle dynamics on all other developing

${ }^{1}$ UPMC Univ Paris 06, UMR 7598, Laboratoire Jacques-Louis Lions, F-75005, Paris, France

CNRS, UMR 7598, Laboratoire Jacques-Louis Lions, F-75005, Paris, France

2 Centre de Recherche INRIA Paris-Rocquencourt, Domaine de Voluceau, B.P. 105 - F-78153 Le Chesnay, France

${ }^{3}$ CMAP, Ecole Polytechnique, CNRS, route de Saclay, F-91128 Palaiseau Cédex-France

(C) EDP Sciences, SMAI 2012 
follicles. The well-posedness of the Cauchy problem is established in 15]. Existence and uniqueness of weak solutions is proved for bounded initial conditions. The competition process was investigated in a game theory approach after reducing the PDE model to coupled ODE systems [14. Control problems associated with this model are investigated in 8 (computation of backwards reachability sets) and 4 (optimal control in minimal time).

We are specifically interested in the numerical issues raised by this multiscale model. In previous works 3,7 , a CTU numerical scheme has been implemented in the Bearclaw ${ }^{1}$ environment, which is based on adaptive mesh refinement using wave-propagation algorithms [12,13. Here, we develop a dedicated code, which allows us (i) to handle the conservative form of the equations, (ii) to deal with the discontinuous coefficients and (iii) to use high performance computing (HPC) techniques in order to speed up the computing and be able to simulate as many as twenty follicles.

The paper is organized as follows. In section 1 , we introduce the biological background and the multiscale model. In section 2, we describe the numerical scheme in detail. In section 3, we illustrate the simulation outputs and assess the algorithm robustness, accuracy and scalability on parallel architectures.

\section{Biological And BiomathematicAl BACKGROUND}

\subsection{Biological background}

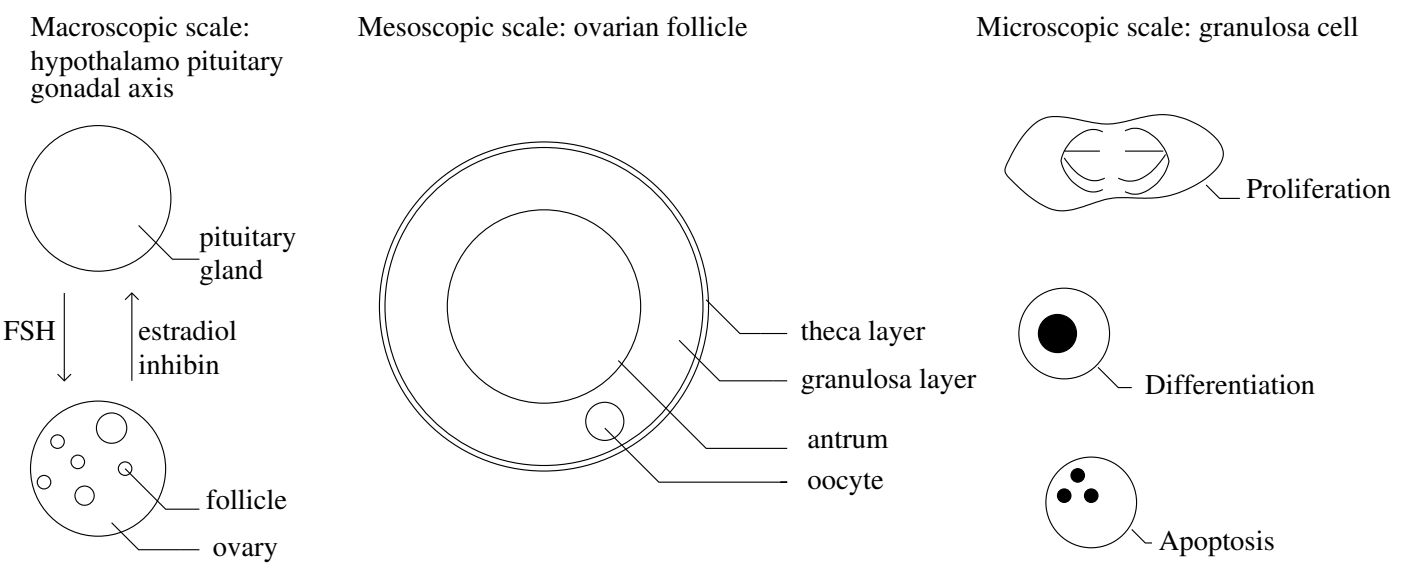

Figure 1. Follicle development as a multiscale process. Left : endocrine feedback loop between the ovaries and the hypothalamo-pituitary-axis. Middle: schematic 2D view of an ovarian follicle. Right : different cell states encountered by the granulosa cells during follicular development.

\subsubsection{Mesoscopic scale: ovarian follicles}

Ovarian follicles are spheroidal tissular structures sheltering the oocytes (figure 1). Each follicle goes through a development process, composed of two main parts : the basal development and the terminal development (figure 2). During basal development, the follicles are independent of hormonal support from the pituitary gland, while, during the terminal part of their development, they become dependent on FSH (follicle stimulating hormone) supply. Follicle development can end up either by ovulation, in the best case, or much more often by degeneration through atresia. The commitment of a follicle to either ovulation or atresia is driven by the changes occurring in the follicular cell population.

\footnotetext{
1 http://www.pas.rochester.edu/ ${ }^{\text {bearclaw/ }}$
} 


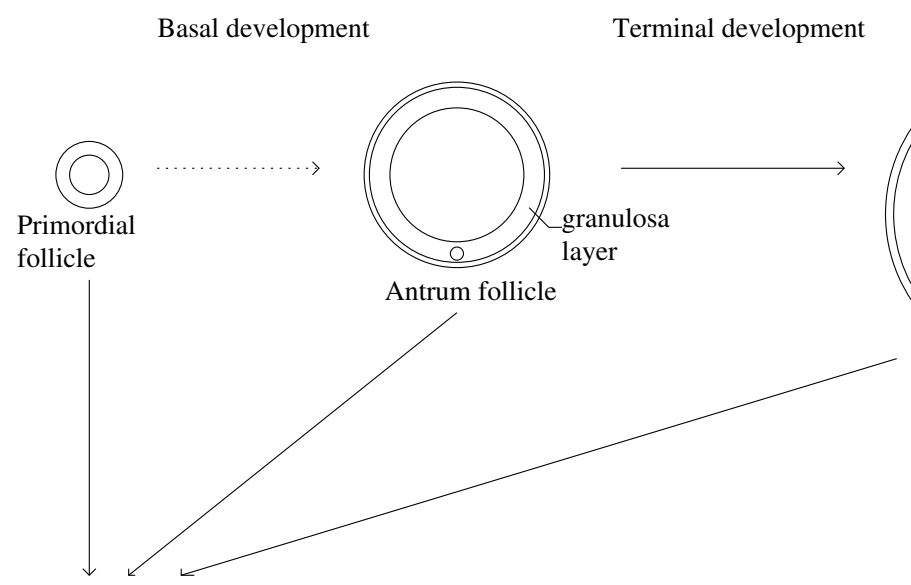

Atresia

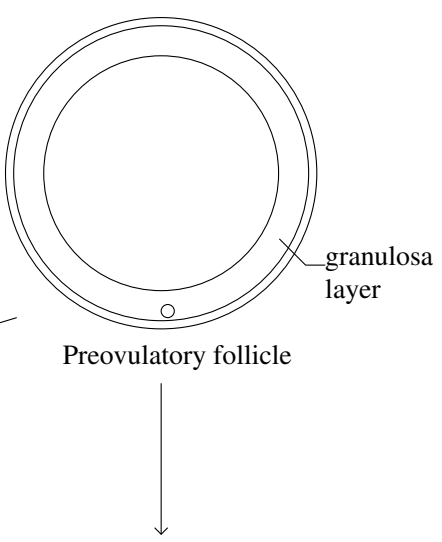

Ovulation

FiguRE 2. Follicular development : from basal to terminal development. After exiting the pool of quiescent primordial follicles, ovarian follicles enter a several-month long process of growth and maturation, that either ends up by ovulation or degeneration through atresia. In the basal part of development, follicle growth is mainly due to the oocyte enlargement and increase in the number of granulosa cell layers. In the final part of development, granulosa cells progressively stop proliferating and follicle growth is mainly due to the enlargement of the antrum, a liquidfilled cavity in the center of the follicle.

\subsubsection{Macroscopic scale : competition process and endocrine feedback loop}

In some sense, the terminal part of follicle development can be considered as a competition between follicles for FSH resource. FSH controls follicle development, while FSH levels are in turn controlled by two hormones secreted by the ovary, inhibin and estradiol, whose plasma levels are determined by the summed contributions of all maturing follicles (figure 1). This ovarian hormonal feedback induces a drop in FSH levels that will penalize the follicles, except those (in the poly-ovulating situations) or that (in the mono-ovulating ones) that are sufficiently mature to survive in a FSH poor environment. The rising levels of estradiol finally triggers the ovulatory surge that leads to the ovulation of the surviving follicles.

\subsubsection{Microscopic scale : granulosa cell kinetics}

Each granulosa cell can be encountered in either of three different cell states : proliferation, differentiation or apoptosis (programmed cell death) (figure 3). At the beginning of terminal development, most granulosa cells are progressing along the cell division cycle, that can be split into a $G 1$ phase, where cells are sensitive to FSH control, and a SM phase, where cells are preparing for mitosis and are insensitive to FSH control. At the end of mitosis, one single mother cell gives birth to two daughter cells. During the FSH sensitive phase, cells become more and more mature, up to a point where they reach a threshold maturity and exit the cell division cycle. At this time they are exposed to a great risk of apoptosis, and may die if the FSH environment is not favorable. After exiting the cell cycle, the cells stop proliferating definitively, but their maturity still increases, so that they contribute more and more to hormone (and especially estradiol) secretion.

\subsection{Biomathematical model}

\subsubsection{Computing domain}

Let us introduce the variables 


$$
\begin{cases}a & \text { age } \\ \gamma & \text { maturity } \\ t & \text { time }\end{cases}
$$

the vector of granulosa cell densities for the $N_{f}$ follicles

$$
\Phi(a, \gamma, t)=\left(\phi_{1}(a, \gamma, t), \ldots, \phi_{N_{f}}(a, \gamma, t)\right),
$$

and the computing domain $\Omega$ in the $(a, \gamma)$ plane,

$$
\Omega=\left\{(a, \gamma), 0 \leq a \leq N_{c} \times D_{a}, 0 \leq \gamma \leq 1\right\}
$$

where $N_{c}$ is the number of cell cycles and $D_{a}$ is the duration of one cycle. The different cell states described in paragraph 1.1 .3 take place in three subdomains as illustrated in Figure 3 . The differentiation phase $D$ corresponds to the area of the domain where the maturity overcomes the cellular maturity threshold $\gamma_{s}$

$$
\left\{\begin{array}{l}
G 1=\left\{(a, \gamma) \in \Omega, \quad p D_{a} \leq a \leq(p+1 / 2) D_{a}, \quad p=0, \ldots, N_{c}-1, \quad 0 \leq \gamma \leq \gamma_{s}\right\} \\
S M=\left\{(a, \gamma) \in \Omega, \quad(p+1 / 2) D_{a} \leq a \leq(p+1) D_{a}, \quad p=0, \ldots, N_{c}-1, \quad 0 \leq \gamma \leq \gamma_{s}\right\} \\
D=\left\{(a, \gamma) \in \Omega, \quad \gamma_{s} \leq \gamma\right\}
\end{array}\right.
$$
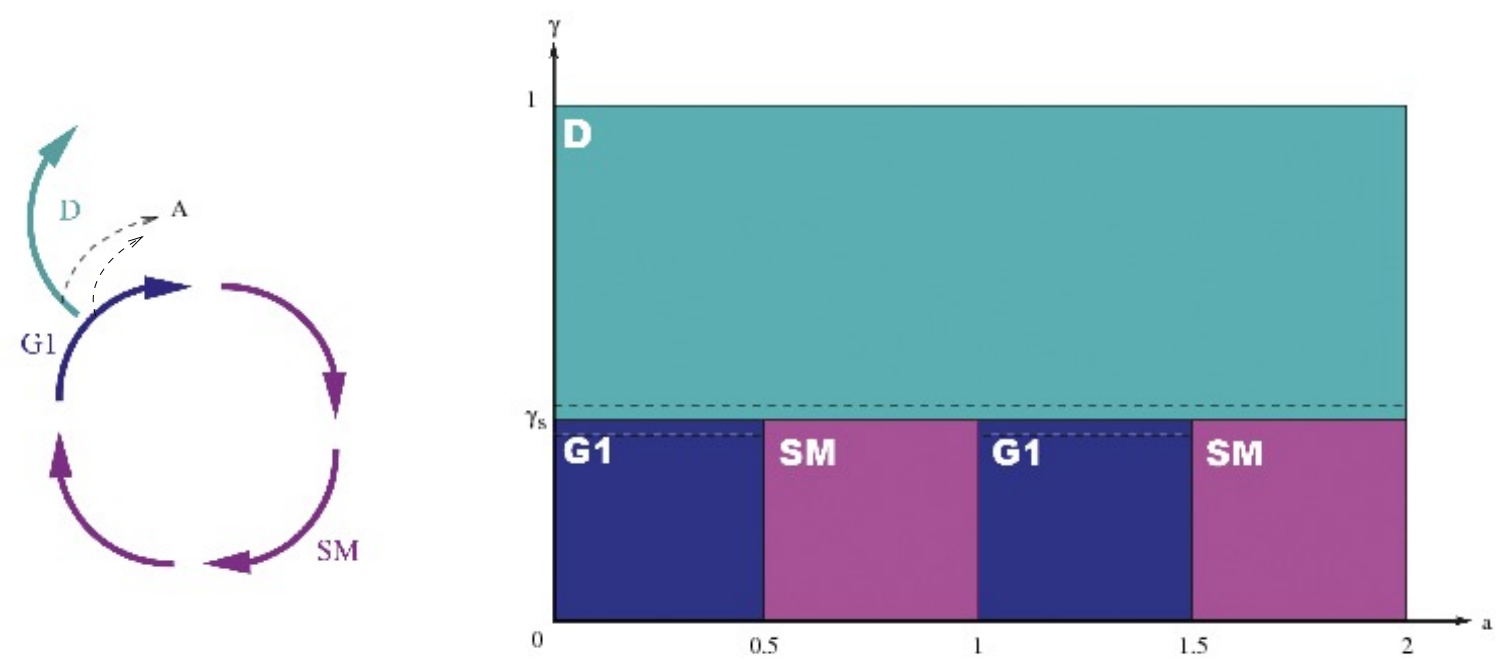

Figure 3. Division of the spatial domain according to the cell phases for unit cycle duration $D_{a}=1$. Left : schematic view of a granulosa cell cycle. Right : $(a, \gamma)$ plane. The bottom part represents the successive cell cycles, each composed of the G1 and SM phases. The top part corresponds to the differentiation phase.

\subsubsection{Hyperbolic system}

If we consider, for each follicle, a conservation law for the granulosa cell population density, we obtain the following hyperbolic system, satisfied by $\Phi$ : 


$$
\begin{cases}\frac{\partial \phi_{1}(a, \gamma, t)}{\partial t}+\frac{\partial\left(g\left(a, \gamma, u_{1}(t)\right) \phi_{1}(a, \gamma, t)\right)}{\partial a}+\frac{\partial\left(h\left(a, \gamma, u_{1}(t)\right) \phi_{1}(a, \gamma, t)\right)}{\partial \gamma} & =-\lambda(a, \gamma, U(t)) \phi_{1}(a, \gamma, t) \\ \frac{\partial \phi_{f}(a, \gamma, t)}{\partial t}+\frac{\partial\left(g\left(a, \gamma, u_{f}(t)\right) \phi_{f}(a, \gamma, t)\right)}{\partial a}+\frac{\partial\left(h\left(a, \gamma, u_{f}(t)\right) \phi_{f}(a, \gamma, t)\right)}{\partial \gamma} & =-\lambda(a, \gamma, U(t)) \phi_{f}(a, \gamma, t) \\ \frac{\partial \phi_{N_{f}}(a, \gamma, t)}{\partial t}+\frac{\partial\left(g\left(a, \gamma, u_{N_{f}}(t)\right) \phi_{N_{f}}(a, \gamma, t)\right)}{\partial a}+\frac{\partial\left(h\left(a, \gamma, u_{N_{f}}(t)\right) \phi_{N_{f}}(a, \gamma, t)\right)}{\partial \gamma} & =-\lambda(a, \gamma, U(t)) \phi_{N_{f}}(a, \gamma, t)\end{cases}
$$

The initial condition is given by the distribution of the granulosa cell populations at the initial time

$$
\Phi(a, \gamma, 0)=\Phi_{0}(a, \gamma)
$$

where $\Phi_{0}$ is compactly supported in $] 0, N_{c} D_{a}[\times] 0,1[$. Since the functional domain $\Omega$ is compact, we need to express boundary conditions. Considering some qualitative properties of the maturation function $h$, defined below, the horizontal top boundary $\gamma=1$ is never reached. Similarly, we can fix the number of cell cycles $N_{c}$ in accordance with the ageing function $g$, so that the right vertical boundary $a=N_{c} D_{a}$ is never reached. For sake of computing simplicity, we therefore assume spatial periodicity on the outer boundaries of $\Omega$ in the numerical simulations.

The ageing function $g$ appearing in (1) is defined by

$$
g(a, \gamma, u)= \begin{cases}g_{1} u+g_{2} & \text { for }(a, \gamma) \in G 1 \\ 1 & \text { for }(a, \gamma) \in S M \cup D\end{cases}
$$

where $g_{1}, g_{2}$ are real positive constants. The maturation function $h$ is defined by

$$
h(a, \gamma, u)= \begin{cases}\tau_{h}\left(-\gamma^{2}+\left(c_{1} \gamma+c_{2}\right)\left(1-\exp \left(\frac{-u}{\bar{u}}\right)\right)\right) & \text { for }(a, \gamma) \in G 1 \cup D \\ 0 & \text { for }(a, \gamma) \in S M\end{cases}
$$

where $\tau_{h}, c_{1}, c_{2}$ and $\bar{u}$ are real positive constants. The source term, that represents cell loss through apoptosis, is defined by

$$
\lambda(a, \gamma, U)= \begin{cases}K \exp \left(-\left(\frac{\left(\gamma-\gamma_{s}\right)^{2}}{\bar{\gamma}}\right)\right) \times(1-U) & \text { for }(a, \gamma) \in G 1 \cup D \\ 0 & \text { for }(a, \gamma) \in S M\end{cases}
$$

where $K, \gamma_{s}$ and $\bar{\gamma}$ are real positive constants.

Remark. The precise values of the constants depends on species and breeds; they will be fixed later.

\subsubsection{Closure equations}

The equations in the PDE system (1) are linked together through the argument $u_{f}(t)$ appearing in the speeds $g\left(a, \gamma, u_{f}\right)$ and $h\left(a, \gamma, u_{f}\right)$ and the argument $U(t)$ in the source term $\lambda(a, \gamma, U)$. $U(t)$ and $u_{f}(t)$ represent respectively the plasma FSH level and the locally bioavailable FSH level and depend on some maturation moments of the densities.

- follicular cell mass (local, one by follicle)

$$
m_{0}(f, t)=\int_{0}^{1} \int_{0}^{N_{c} D_{a}} \phi_{f}(a, \gamma, t) \mathrm{d} a \mathrm{~d} \gamma, \quad 1 \leq f \leq N_{f}
$$

This moment is an interesting quantity since it corresponds to an observable variable (the total cell number in one follicle) even if it does not come as such into play in the equations. 
- follicular maturity (local, one by follicle)

$$
m(f, t)=\int_{0}^{1} \int_{0}^{N_{c} D_{a}} \gamma \phi_{f}(a, \gamma, t) \mathrm{d} a \mathrm{~d} \gamma, \quad 1 \leq f \leq N_{f}
$$

- ovarian maturity (global, shared by all follicles)

$$
M(t)=\sum_{f=1}^{N_{f}} m(f, t),
$$

- plasma FSH level (global, shared by all follicles)

$$
U(t)=U_{\min }+\frac{1-U_{\min }}{1+\exp (c(M(t)-\bar{M}))},
$$

where $U_{\min }, c$ and $\bar{M}$ are real positive constants

- locally bioavailable FSH level

$$
u_{f}(t)=\min \left(b_{1}+\frac{e^{b_{2} m(f, t)}}{b_{3}}, 1\right) U(t),
$$

where $b_{1}, b_{2}$ and $b_{3}$ are real positive constants.

It is important to notice that the ageing function (2) is discontinuous (in general) on the interfaces $G 1-S M$, and that the maturation function (3) is discontinuous (in general) on the interfaces $S M-D$ (Figure 3 ). It is necessary to introduce transmission conditions in order to overcome possible failure of uniqueness due to the discontinuities in coefficients (see [10] or [1] for instance). The precise definition of the required transmission conditions has been addressed in the paper by Peipei Shang ( [15]). We suppose that, for each cycle $p=1, \ldots, N_{c}$ and each follicle $f=1, \ldots, N_{f}$, the flux on the $a$-axis is continuous between the phases $G 1$ and $S M$

$$
\phi_{f}\left(t, a^{+}, \gamma\right)=\left(g_{1} u_{f}+g_{2}\right) \phi_{f}\left(t, a^{-}, \gamma\right), \quad a=(p-1 / 2) D_{a}, \quad 0 \leq \gamma \leq \gamma_{s},
$$

and that the flux is doubling on the interfaces $S M-G 1$, which accounts for the birth of new cells at the end of each cell cycle

$$
\left(g_{1} u_{f}+g_{2}\right) \phi_{f}\left(t, a^{+}, \gamma\right)=2 \phi_{f}\left(t, a^{-}, \gamma\right), \quad a=p D_{a}, \quad 0 \leq \gamma \leq \gamma_{s} .
$$

Finally, we suppose homogeneous Dirichlet condition to the north of the interface $S M-D$

$$
\phi_{f}\left(t, a, \gamma_{s}^{+}\right)=0, \quad(p-1 / 2) D_{a} \leq a \leq p D_{a} .
$$

The time dependent ovarian maturity $M(t)$ defined in (7) is compared to the ovarian maturity threshold, denoted by $M_{o}$, to define the final time, beyond which the competition is over

$$
t_{\text {final }}=\inf \left\{t, M(t) \geq M_{o}\right\} .
$$

The follicles are then sorted into two classes : ovulatory if the maturity is higher than the follicle maturity threshold, denoted by $M_{f}$, atretic otherwise.

\section{Numerical Method}

\subsection{Discretization}

In this paragraph the cycle duration is set to $D_{a}=1$. We denote by $\Delta a$ (respectively $\Delta \gamma$ ) the space step in the age (respectively maturity) direction. In practice we choose $\Delta a=\Delta \gamma$. The discretization step $\Delta \gamma$ 
and the cellular maturity threshold $\gamma_{s}$ must be chosen so that the interfaces where the speed coefficients $(2 \mid 3)$ are discontinuous fall on grid points covering the computational domain of size $\left[N_{c}, 1\right]$. Denoting by $N_{m}$ the number of grid cells by half granulosa cell cycle, we set $N_{\gamma}=2 N_{m}$ and $\Delta a=\Delta \gamma=1 / N_{\gamma}$ and we introduce the dedicated ${ }^{2}$ notations for grid points $\left(a_{k}, \gamma_{l}\right)$ and mesh centers $\left(a_{k+1 / 2}, \gamma_{l+1 / 2}\right)$

$$
\begin{aligned}
& a_{k}=k \Delta a, \quad a_{k+1 / 2}=(k+1 / 2) \Delta a, \text { for } k=0, \ldots, N_{c} \times N_{\gamma}, \\
& \gamma_{l}=l \Delta \gamma, \quad \gamma_{l+1 / 2}=(l+1 / 2) \Delta \gamma, \text { for } l=0, \ldots, N_{\gamma} .
\end{aligned}
$$

Considering that the time step $\Delta t^{n}$ may change at every iteration, in order to preserve stability, the time discretization is defined by

$$
t^{0}=0, \quad t^{n+1}=t^{n}+\Delta t^{n}, \quad \text { for } n=0, \ldots, N_{t}
$$

with $N_{t}$ such that $t^{N_{t}}=t_{\text {final }}$. The unknowns are the approximate mean values of the density vector in each grid mesh

$$
\Phi_{k, l}^{n} \approx \frac{1}{\Delta a \Delta \gamma} \int_{a_{k}}^{a_{k+1}} \int_{\gamma_{l}}^{\gamma_{l+1}} \Phi\left(a, \gamma, t^{n}\right) d \gamma d a, \quad \text { for } \quad k=0, \ldots, N_{c} N_{\gamma}-1, \text { and } l=0, \ldots, N_{\gamma}-1
$$

whose components $\phi_{f, k, l}^{n}$ are the discrete density values for each follicle.

\subsection{Macroscopic scale : piecewise constant approximation of the hormonal control}

We define the approximation of the control terms (5-9) at each time step $n=0, \ldots, N_{t}$

$$
\begin{aligned}
m_{0, f}^{n} & =\Delta a \Delta \gamma \sum_{l=0}^{N_{\gamma}-1} \sum_{k=0}^{N_{c} N_{\gamma}-1} \phi_{f, k, l}^{n}, \quad \text { for } f=1, \ldots, N_{f}, \\
m_{f}^{n} & =\Delta a \Delta \gamma \sum_{l=0}^{N_{\gamma}-1} \gamma_{l+1 / 2} \sum_{k=0}^{N_{c} N_{\gamma}-1} \phi_{f, k, l}^{n}, \quad \text { for } f=1, \ldots, N_{f}, \\
M^{n} & =\sum_{f=1}^{N_{f}} m_{f}^{n}, \\
U^{n} & =U_{\min }+\frac{1-U_{\min }}{1+\exp \left(c\left(M^{n}-\bar{M}\right)\right)}, \\
u_{f}^{n} & =\min \left(b_{1}+\frac{e^{b_{2} m_{f}^{n}}}{b_{3}}, 1\right) U^{n}, \quad \text { for } f=1, \ldots, N_{f} .
\end{aligned}
$$

\subsection{Mesoscopic scale : finite volume scheme}

We use a splitting strategy in order to compute the solution of the PDE system (1), which amounts to a convective equation combined with a source equation, for each follicle $f=1, \ldots, N_{f}$

$$
\begin{aligned}
\partial_{t} \phi_{f}(a, \gamma, t)+\partial_{a}\left(g\left(a, \gamma, u_{f}(t)\right) \phi_{f}(a, \gamma, t)\right)+\partial_{\gamma}\left(h\left(a, \gamma, u_{f}(t)\right) \phi_{f}(a, \gamma, t)\right)=0 & \text { (convective part) }, \\
\partial_{t} \phi_{f}(a, \gamma, t)=-\lambda(a, \gamma, U(t)) \phi_{f}(a, \gamma, t) & \text { (source part). }
\end{aligned}
$$

\footnotetext{
${ }^{2}$ We use this index notation for the use of the forthcoming Multiresolution development
} 


\subsubsection{Convective part}

The convection part 22 is treated with a classical finite volume method. The approximate mean values of the solution at $t=0$ are initialized using a midpoint formula, accurate at the order 2 in space

$$
\Phi_{k, l}^{0}=\Phi_{0}\left(a_{k+1 / 2}, \gamma_{l+1 / 2}\right) .
$$

Using the integral form of the conservation law

$$
\int_{t^{n}}^{t^{n+1}} \int_{a_{k}}^{a_{k+1}} \int_{\gamma_{l}}^{\gamma_{l+1}}\left(\partial_{t} \phi_{f}(a, \gamma, t)+\partial_{a}\left(g\left(a, \gamma, u_{f}(t)\right) \phi_{f}(a, \gamma, t)\right)+\partial_{\gamma}\left(h\left(a, \gamma, u_{f}(t)\right) \phi_{f}(a, \gamma, t)\right)\right) d \gamma d a d t=0
$$

we obtain a recursion on the approximate density of each follicle, where we drop the index $f$ for clarity sake

$$
\phi_{k, l}^{n+1}=\phi_{k, l}^{n}-\frac{\Delta t}{\Delta a}\left(G_{k+1, l+\frac{1}{2}}\left(\Phi^{n}\right)-G_{k, l+\frac{1}{2}}\left(\Phi^{n}\right)\right)-\frac{\Delta t}{\Delta \gamma}\left(H_{k+\frac{1}{2}, l+1}\left(\Phi^{n}\right)-H_{k+\frac{1}{2}, l}\left(\Phi^{n}\right)\right)
$$

where $G_{k, l+\frac{1}{2}}$ (respectively $\left.H_{k+\frac{1}{2}, l}\right)$ is the numerical flux across the vertical edge $\left[\left(a_{k}, \gamma_{l}\right),\left(a_{k}, \gamma_{l+1}\right)\right]$ (respectively the horizontal edge $\left.\left[\left(a_{k}, \gamma_{l}\right),\left(a_{k+1}, \gamma_{l}\right)\right]\right)$ defined by

$$
\left\{\begin{array}{l}
G_{k, l+\frac{1}{2}}\left(\Phi^{n}\right) \approx \frac{1}{\Delta t^{n} \Delta \gamma} \int_{t^{n}}^{t^{n+1}} \int_{\gamma_{l}}^{\gamma_{l+1}} g\left(a_{k}, \gamma, u_{f}(t)\right) \phi\left(a_{k}, \gamma, t\right) \mathrm{d} \gamma \mathrm{d} t \\
H_{k+\frac{1}{2}, l}\left(\Phi^{n}\right) \approx \frac{1}{\Delta t^{n} \Delta a} \int_{t^{n}}^{t^{n+1}} \int_{a_{k}}^{a_{k+1}} h\left(a, \gamma_{l}, u_{f}(t)\right) \phi\left(a, \gamma_{l}, t\right) \mathrm{d} a \mathrm{~d} t
\end{array}\right.
$$

The dependence of the flux functions on all the follicle densities through the control term $u_{f}$ is emphasized by the $\Phi^{n}$ argument. It appears in the explicit in time approximations of the speeds (2), and (3) at the center of the mesh

$$
\left\{\begin{array}{l}
g_{k, l}^{n}=g\left(a_{k+\frac{1}{2}}, \gamma_{l+\frac{1}{2}}, u_{f}^{n}\right) \\
h_{k, l}^{n}=h\left(a_{k+\frac{1}{2}}, \gamma_{l+\frac{1}{2}}, u_{f}^{n}\right)
\end{array}\right.
$$

Note that the transmission conditions $\sqrt{10}$ and $\sqrt{11}$ on the interfaces where these speed coefficients $(2)$ and $(3)$ are discontinuous are exactly treated with the method described in the article of Godlewski and Raviart ( 10$]$ ). The numerical fluxes (25) are designed using a limiter strategy. Indeed, it is well known that first order schemes, like the Godunov scheme, are diffusive, and that second order schemes, like Lax Wendroff scheme, generate oscillations in the neighborhood of discontinuities. In order to get a stable as well as precise scheme, we take a weighting of a low order scheme and a high order scheme, and we define limited numerical fluxes

$$
\begin{gathered}
G=G^{\text {Low }}+\ell\left(r_{G}\right)\left(G^{H i g h}-G^{\text {Low }}\right), \\
H=H^{\text {Low }}+\ell\left(r_{H}\right)\left(H^{H i g h}-H^{\text {Low }}\right),
\end{gathered}
$$

where $\ell$ is a limiter function, for example van Leer function

$$
\ell(r)=\frac{r+|r|}{1+r}
$$

and $r_{G}, r_{H}$ are

$$
r_{G}=\left\{\begin{array}{l}
\frac{g_{k-1, l} \phi_{k-1, l}-g_{k-2, l} \phi_{k-2, l}}{g_{k, l} \phi_{k, l}-g_{k-1, l} \phi_{k-1, l}} \quad \text { if } \quad g_{k-2, l} \geq 0 \quad \text { and } \quad g_{k-1, l} \geq 0 \quad \text { and } \quad g_{k, l} \geq 0, \\
\frac{g_{k+1, l} \phi_{k+1, l}-g_{k, l} \phi_{k, l}}{g_{k, l} \phi_{k, l}-g_{k-1, l} \phi_{k-1, l}} \quad \text { if } \quad g_{k-1, l} \leq 0, \quad \text { and } \quad g_{k, l} \leq 0 \quad \text { and } \quad g_{k+1, l} \leq 0, \\
0 \quad \text { otherwise, }
\end{array}\right.
$$




$$
r_{H}=\left\{\begin{array}{l}
\frac{h_{k, l-1} \phi_{k, l-1}-h_{k, l-2} \phi_{k, l-2}}{h_{k, l} \phi_{k, l}-h_{k, l-1} \phi_{k, l-1}} \quad \text { if } \quad h_{k, l-2} \geq 0 \quad \text { and } \quad h_{k, l-1} \geq 0 \quad \text { and } h_{k, l} \geq 0, \\
\frac{h_{k, l+1} \phi_{k, l+1}-h_{k, l} \phi_{k, l}}{h_{k, l} \phi_{k, l}-h_{k, l-1} \phi_{k, l-1}} \quad \text { if } \quad h_{k, l-1} \leq 0 \quad \text { and } \quad h_{k, l} \leq 0 \quad \text { and } \quad h_{k, l+1} \leq 0, \\
0 \quad \text { otherwise. }
\end{array}\right.
$$

These ratios are good indicators of the regularity of the function in each direction (see [16]). In fact, a steep gradient or a discontinuity gives a ratio far from 1, whereas a smooth function gives a ratio close to 1 . The first order fluxes entering equation (26) are the Godunov fluxes

$$
\left\{\begin{array}{l}
G_{k, l+\frac{1}{2}}^{L o w}\left(\Phi^{n}\right)=\left(g_{k-1, l}^{n}\right)^{+} \phi_{k-1, l}^{n}+\left(g_{k, l}^{n}\right)^{-} \phi_{k, l}^{n}, \\
H_{k+\frac{1}{2}, l}^{L o w}\left(\Phi^{n}\right)=\left(h_{k, l-1}^{n}\right)^{+} \phi_{k, l-1}^{n}+\left(h_{k, l}^{n}\right)^{-} \phi_{k, l}^{n}
\end{array}\right.
$$

and the high order fluxes are the Lax Wendroff ones

$$
\left\{\begin{array}{l}
G_{k, l+\frac{1}{2}}^{H i g h}\left(\Phi^{n}\right)=\frac{1}{2}\left(g_{k-1, l}^{n} \phi_{k-1, l}^{n}+g_{k, l}^{n} \phi_{k, l}^{n}\right), \\
H_{k+\frac{1}{2}, l}^{H i g h}\left(\Phi^{n}\right)=\frac{1}{2}\left(h_{k, l-1}^{n} \phi_{k, l-1}^{n}+h_{k, l}^{n} \phi_{k, l}^{n}\right) .
\end{array}\right.
$$

Consequently, since $\Delta a=\Delta \gamma$, the CFL condition which guarantees the stability is

$$
\Delta t_{f, F V S}^{n} \leq C F L \frac{\Delta \gamma}{\max _{k, l}\left(\left|g_{k, l}\right|,\left|h_{k, l}\right|\right)}
$$

with $C F L \leq \frac{1}{2}$.

\subsubsection{Source part}

The source part 230 of the PDE system is explicitly dealt with

$$
\phi_{k, l}^{n+1}=\phi_{k, l}^{n}-\Delta t \lambda\left(a_{k+\frac{1}{2}}, \gamma_{l+\frac{1}{2}}, U^{n}\right) \phi_{k, l}^{n},
$$

which implies a stability condition on the time step, which we strengthen to enforce positivity

$$
\Delta t_{f, \text { source }}^{n} \leq \frac{1}{\max _{k, l}\left|\lambda\left(a_{k+\frac{1}{2}}, \gamma_{l+\frac{1}{2}}, U^{n}\right)\right|} .
$$

\subsection{Macro/Meso scale Parallelization}

In order to speed up the numerical simulations, the code is implemented on a parallel architecture. Every follicle follows its own dynamic independently of the others, except that it shares with them the FSH resource, and that it contributes to the tuning of plasma FSH level. Hence it is natural to use a SIMD (single instruction, multiple data) strategy, with one follicle by process. The only communications that have to be done at each time step concern the ovarian maturity, through a reduction operation (sum) and the current time step $\Delta t^{n}$, through a reduction operation $(\mathrm{min})$. Each process computes the maximum time step satisfying both conditions (27) and (29)

$$
\Delta t_{f}^{n}=\min \left\{\Delta t_{f, F V S}^{n}, \Delta t_{f, \text { source }}^{n}\right\}
$$

relevant for its own follicle, and the time step used by all processes is

$$
\Delta t^{n}=\min _{f}\left\{\Delta t_{f}^{n}\right\} .
$$




\subsection{Parallel algorithm and improvement to order 2 in time}

Finally, we get the following parallel algorithm for each time step:

(1) Compute time step

- Compute local time step $\Delta t_{f}^{n}(30$

- Communication : Compute the minimum of local time steps $\Delta t^{n}$

(2) Update solution

- Flux computation 26,

- Convection computation (24),

- Source computation (28),

(3) Update control terms

- Compute local maturity (18),

- Communication : compute the sum of local maturities (19),

- Compute FSH plasma level (20),

- Compute locally bioavailable FSH rate (21).

Denoting by $E$ the evolution operator, that consists in steps (2) and (3), the second order in time is achieved by a second order Runge-Kutta method (Heun)

$$
\begin{aligned}
\phi^{*} & =E\left(\phi^{n}\right), \\
\phi^{* *} & =E\left(\phi^{*}\right), \\
\phi^{n+1} & =\frac{1}{2}\left(\phi^{n}+\phi^{* *}\right) .
\end{aligned}
$$

\section{Numerical Simulations}

The code was developed in $\mathrm{C}++$ using the parallel library MPI. The tests were performed on the JacquesLouis Lions Laboratory super calculator SGI Altix UV 100. The current configuration is 64-core 2 GHz. The visualizations were made with OpenGL and gnuplot.

\subsection{Initial condition}

In the first test case, we used, for sake of comparison with anterior results, a piecewise constant function similar to the initial condition used in 7,8]. Its support in age covers the first cycle while its amplitude is a step-decreasing function of the maturity.

$$
\phi_{0}(a, \gamma)=\left\{\begin{array}{c}
8 \text { if } 0.05 \leq \gamma<0.1 \\
7 \text { if } 0.1 \leq \gamma<0.15 \\
5 \text { if } 0.15 \leq \gamma<0.2 \\
0 \text { otherwise. } \\
0 \text { otherwise. }
\end{array}\right\} \text { if } 0 \leq a \leq 1
$$

For the other test cases we used as initial condition a gaussian function centered in $C=\left(C_{a}, C_{\gamma}\right)$

$$
\phi_{0}(a, \gamma)=\frac{1}{2 \pi \sigma} \exp \left(\frac{1}{2}\left(\frac{\left(a-C_{a}\right)^{2}}{\sigma^{2}}+\frac{\left(\gamma-C_{\gamma}\right)^{2}}{\sigma^{2}}\right)\right)
$$

The variance $\sigma^{2}$ can be chosen so that $\phi_{0}$ be smooth enough to be used for testing the convergence rate, which is checked in the paragraph 3.5. 


\subsection{Sets of default parameters}

Since one of our goals was to improve the computational method, we used the same set of parameters as in 8 to be able to compare at least qualitatively our results to previous simulation outputs. We distinguished two sets of parameters, one, in Table 1 , for the global parameters, that are identical for all follicles (space discretization, CFL condition,...), another, in Table 2, for the local parameters (initial condition, velocity parameters,...), which can depend on the follicle.

\begin{tabular}{|c|c|c|}
\hline Parameter & Description & Value \\
\hline$N_{m}$ & number of grid cells by half granulosa cell cycle & 30 \\
$N_{c}$ & number of cycles & 8 \\
CFL & CFL condition & 0.4 \\
\hline$M_{f}$ & follicular maturity threshold & 0.7 \\
$M_{o}$ & ovarian maturity threshold & 5. \\
\hline & FSH plasma level (eq. [8) $)$ & \\
$U_{\min }$ & minimum level & 0.075 \\
$\bar{M}$ & slope parameter & 2.0 \\
$\bar{M}$ & abscissa of the inflection point & 4.5 \\
\hline & Apoptosis source term (eq. 44) & \\
$K$ & intensity factor & 6.0 \\
$\bar{\gamma}$ & scaling factor & 0.02 \\
\hline$t_{\max }$ & maximum time (to avoid excessively long computations) & 7 \\
\hline
\end{tabular}

TABLE 1. Values of the global parameters used in Figures 4 to 9

. The number of follicles, $N_{f}$, and the number of grid cells by half granulosa cell cycle, $N_{m}$, may depend on the simulation.

\begin{tabular}{|c|c|c|}
\hline Parameter & Description & Value \\
\hline$\gamma_{s}$ & cellular maturity threshold & 0.3 \\
\hline$b_{1}$ & intrafollicular FSH level (eq. (9)) & \\
$b_{2}$ & basal level & 0.08 \\
$b_{3}$ & exponential rate & 2.25 \\
& scaling factor & 1450. \\
$g_{1}$ & aging function (eq. (2)) & \\
$g_{2}$ & origin & 4.0 \\
& Maturation function (eq. & 1.2 \\
$\tau_{h}$ & ab) & 1 \\
$c_{1}$ & abscissa of the center & 0.68 \\
$c_{2}$ & ordinate of the center & 0.08 \\
$\bar{u}$ & variance & 0.02 \\
\hline & $C_{a}$ & 0.15 \\
$C_{\gamma}$ & $\sigma^{2}$ & 0.0025 \\
\hline
\end{tabular}

TABLE 2. Values of the local (follicle-dependent) parameters used in Figures 4 to 9 


\subsection{Time evolution of the cell density for one follicle}

We have first studied the behavior of the cell density of one single follicle as a function of time, in order to check some qualitative properties.

The full movie can be downloaded from http://www.ljll.math.upmc.fr/aymard/CEMRACS2011.gif and four snapshots are displayed on Figure 4 Note that on the snapshots the color code is time dependent while in the movie it is set once and for all at initial time.

The parameters values are gathered in Table 1 and 2 , except for $g_{2}=1.2$ and $\tau_{h}=1.2$. Also, in this monoovulatory situation we set the follicular and ovarian maturity thresholds equal to $M_{o}=M_{f}=16.5$. The spatial discretization for this test uses $N_{m}=30$ cells per half granulosa cell cycle, and the domain $\Omega$ contains eight cycles. The grid size is therefore $8 \times(2 \times 30)^{2}=28800$.

On the first snapshot 4 a), we can observe the piecewise constant initial condition (32). The cell mass is equal to 1.

On the second snapshot $4 \mathrm{~b}$ ), at time 0.91 , we can see that the density has moved to the second cycle. The cell mass is equal to 2.17. At the interface $a=2.5$ between zone $G 1$ and $S M$ the ageing function decreases, which results in a local increase in the density, whose profile becomes narrower. The cell density is starting to double at the end of the second cell cycle, on the $S M-G 1$ interface at $a=3$.

On the third snapshot 4 c), at time 1.57, the cell density has reached the third cycle. It is splitting into a fully differentiated subpopulation and a still proliferating one. It is worth noticing that, consistently with the model, there is no crossing of the $S M-D$ interface. The cell mass is equal to 3.7 .

On the last snapshot $4 \mathrm{~d}$ ), around time $t=5$, towards the end of the simulation, the density is concentrated in the $D$ phase above the seventh cell cycle. Even if all cells have exited the cell cycle, we can distinguish three different density clouds, each of which being issued from one of the previous cycles. The cell mass is equal to 14.7.

In Figure 5 the time evolution of the cell mass (panel A) and follicular maturity (panel B) are displayed as green curves. While the cell mass reaches a constant value as soon as all cells have entered the differentiating phase, the maturity goes on increasing. The blue curves correspond to a similar simulation with a slower maturation function, where $\tau_{h}=1$ instead of 1.2. The cells spend more time in the proliferating phase and they enter the differentiating phase at a later time. Since more mitosis have happened, the cell mass reaches a higher value, around 25 instead of 14.7 .

In the first case, with the faster maturity function, the final time condition defined by $(13)$ is not reached and the simulation is stopped at $t_{\max }=7$ after 1967 time steps. This is more than the time required to cover the eight cell cycles. Yet at that time, the follicular maturity, displayed on panel B of Figure 5, is around 11. far below the follicular maturity threshold of $M_{f}=16.5$. In the second case the follicular maturity overcomes the follicular maturity threshold at time $t=6.85$ and is around 17 . at the end of the simulation.

This behavior of the numerical solution is in accordance with the previous simulations presented in $[8]$ and $[7]$.

\subsection{Competition between ten follicles}

The second test consists in a simulation of a competition process between ten follicles. The parameters defining the plasma FSH level are set to $U_{\min }=0.9$ and $c=10$ and the intensity of the source term is set to $K=1$. The follicles are distinguished by the parameter defining their ageing rate (2) at the origin. We consider a range of $g_{2}$ values running from 0.5 to 0.95 , with a 0.05 increment from one follicle to the other

$$
g_{2}=0.5,0.55, \ldots, 0.95 \text {. }
$$

The maximum time is set to $t_{\max }=1.5$. For all other parameters we use the values in Tables 1 and 2, Figure 6 represents a) the plasma FSH level $U(t)$ defined by (8D b) the locally bioavailable FSH level $u_{f}(t)$ defined by (9) c) the cell mass $m_{0}(f, t)$ of each follicle defined by (5), d) the ovarian maturity $M(t)$ defined by (7) and e) the follicular maturity $m(f, t)$ defined by (6). Figure 6. d) shows that the ovarian maturity reaches the threshold defined by (13) with $M_{o}=5$ around $t=1.2$. 


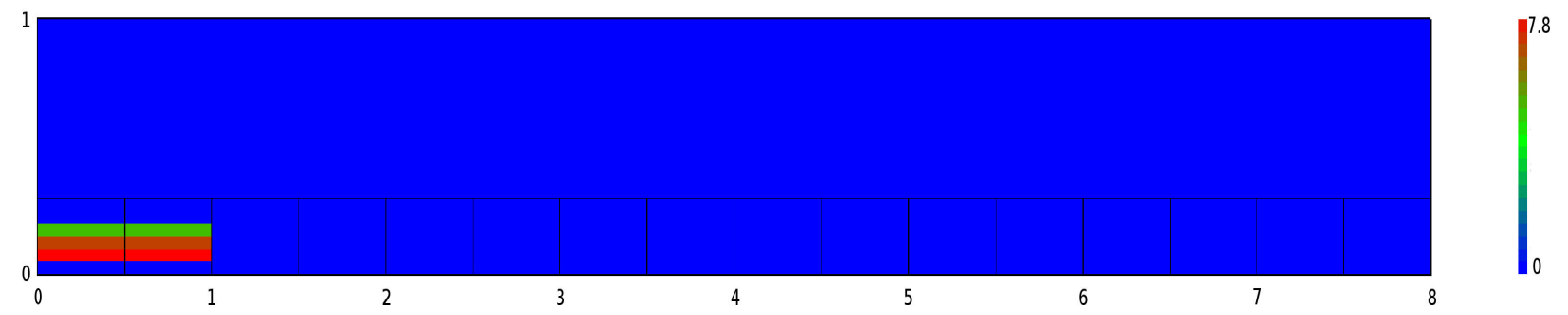

a) Initial condition. Piecewise constant in $\gamma$ and constant in $a$ in the first cell cycle.

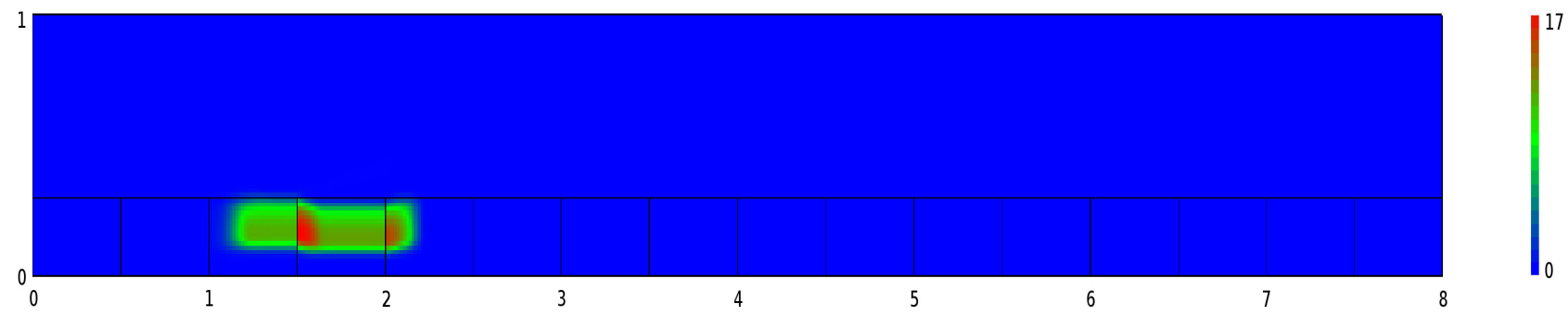

b) Local increase in the density due to the decrease in the ageing function at the interface between the $G 1$ and $S M$ phases.

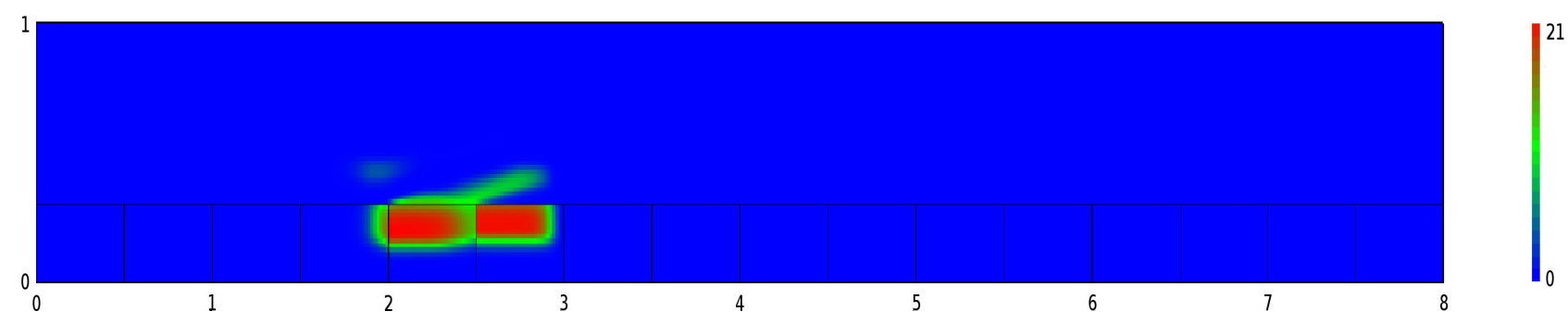

c) Doubling of the cell density at the end of the second cycle and partial transfer into the differentiation phase.

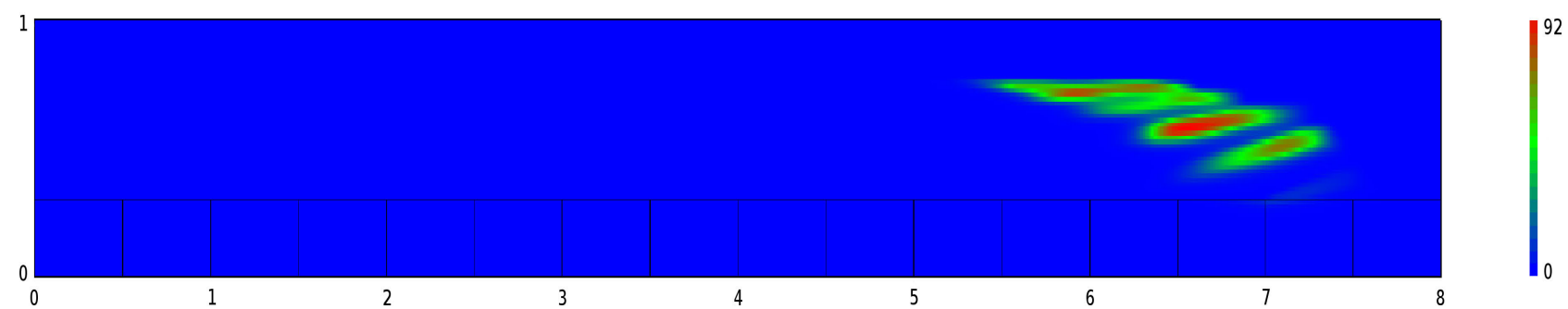

d) Final density consisting of several clouds of fully differentiated cells.

FiguRE 4. Snapshots of the cell density at different times, starting from a piecewise constant density in the first cell cycle. The color code is time dependent. The full movie is available at http://www.ljll.math.upmc.fr/aymard/CEMRACS2011.gif

Figure 6. e) shows that, at the final time, only three follicles have reached the ovulatory stage, where the follicle maturity defined by 6 is higher than $M_{f}=0.7$. They correspond to the values of the parameter $g_{2}=0.5,0.55$ and 0.6 . 

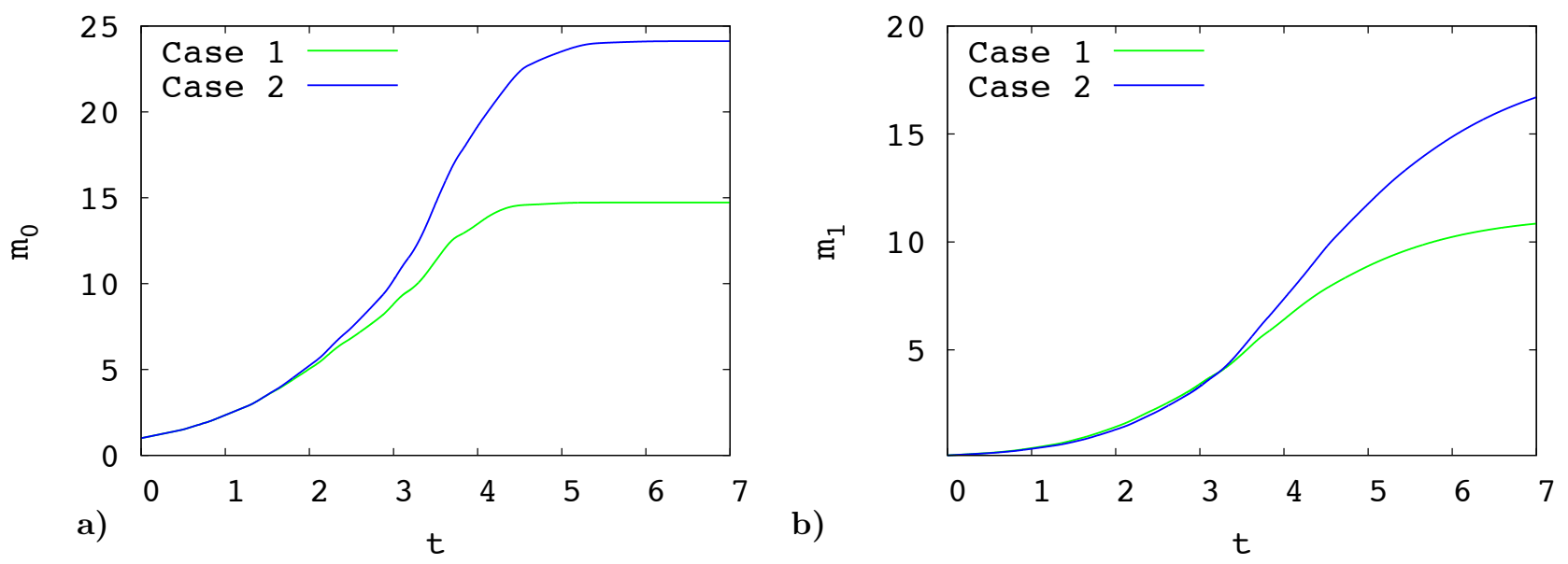

Figure 5. a) Cell mass and b) follicular maturity time evolution for two single follicles. Case 1 corresponds to the parameters used in Figure 4 . Case 2 differs from case 1 by the value of the time constant of the maturity function $\left(\tau_{h}=1\right.$.)

This setup will be used in future work to mimic realistic configurations where ten to twenty follicles can interact together. However the calibration of the model is still in its preliminary stage and the parameters used in this simulation do not meet exactly the biological specifications such as the number of cell cycles performed before ovulation or the final cell number in ovulatory follicles.

\subsection{Convergence rate test}

We now turn to the validation of the code, which consists in numerically verifying the asymptotic order of convergence when the time step $\Delta t$ and space discretization step $\Delta \gamma$ go to zero. We use the parameters in Tables 1 and 2, except for the gaussian function, whose variance is set to $\sigma^{2}=0.002$, so that it is very smooth. The simulation is stopped at $t_{\max }=0.05$, which allows us to reduce the number of cell cycles to $N_{c}=1$ and to discretize the solution on a square grid $N_{\gamma} \times N_{\gamma}$. We compute the solution for six different levels discretizations

$$
N_{\gamma}=80,160,320,640,1280,2560
$$

with the time discretization provided by the stability condition 30 .

\subsubsection{Convergence of the numerical scheme for the linear transport}

We first study the convergence of the numerical scheme when the initial condition is centered in the $S M$ phase $C=(0.7,0.15)$ and the final time is small enough for the density to remain in this phase. The maturity function (3) and the source term (4) are both null, and the ageing function (2) is constant and equal to one. For such a constant linear transport, we can compute the exact solution at the final time $t^{N_{t}}=t_{\max }$ using the characteristic method as well as the error in $L_{1}$-norm of the numerical solution

$$
E(\Delta \gamma)=\Delta \gamma^{2} \sum_{k=0}^{N_{\gamma}-1} \sum_{l=0}^{N_{\gamma}-1}\left|\phi_{k, l}^{N_{t}}-\phi_{0}\left(a_{k+\frac{1}{2}}-t^{N_{t}}, \gamma_{l+\frac{1}{2}}\right)\right| .
$$

The error curve in log scale is superposed with the theoretical order $O\left(\Delta \gamma^{2}\right)$ in Figure 7 . 


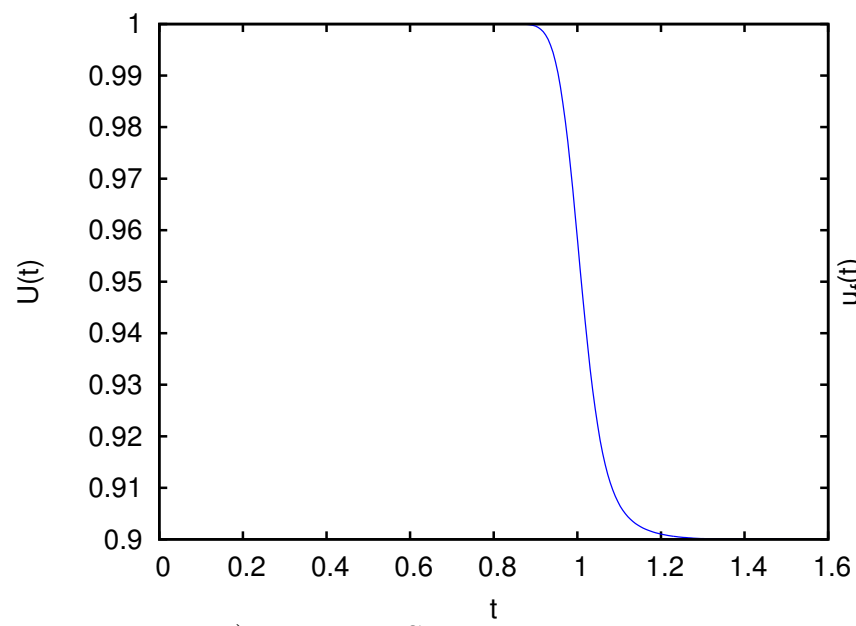

a) Plasma FSH level

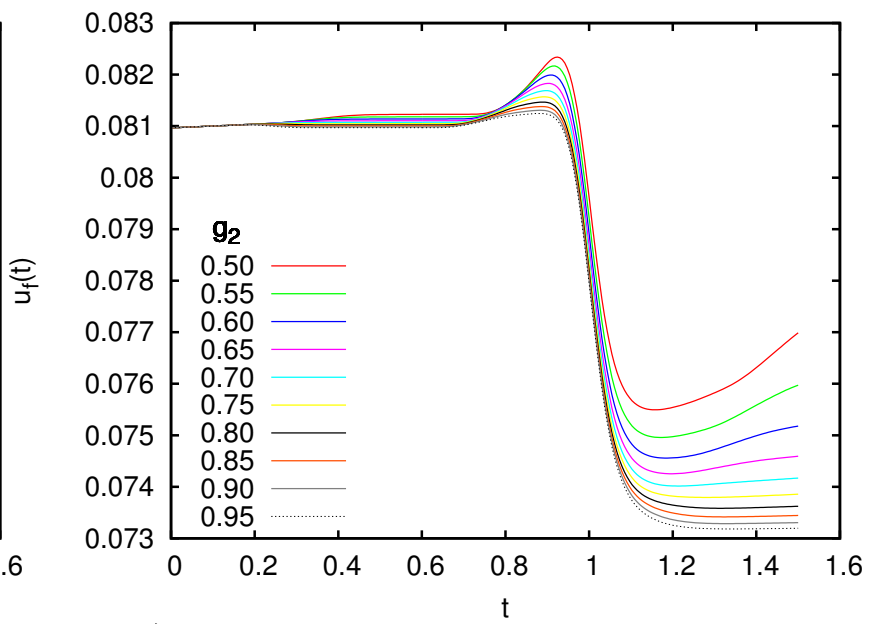

b) Locally bioavailable FSH level

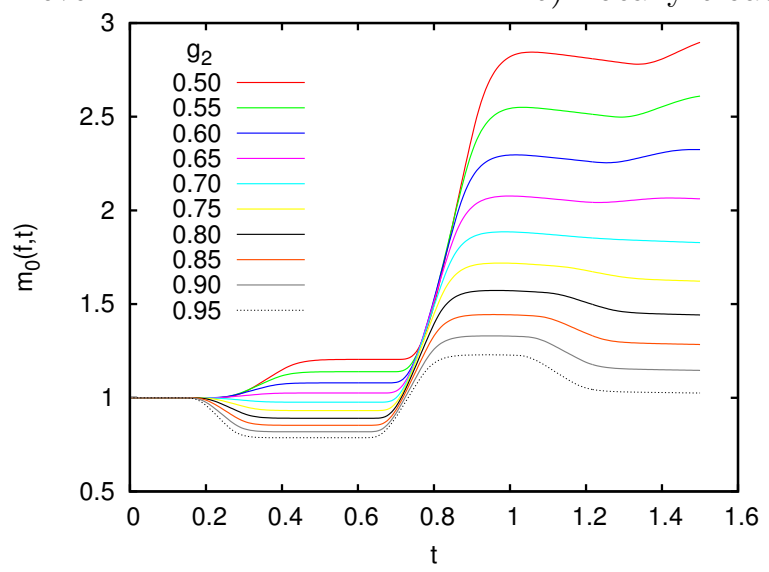

c) Cell mass of each follicle

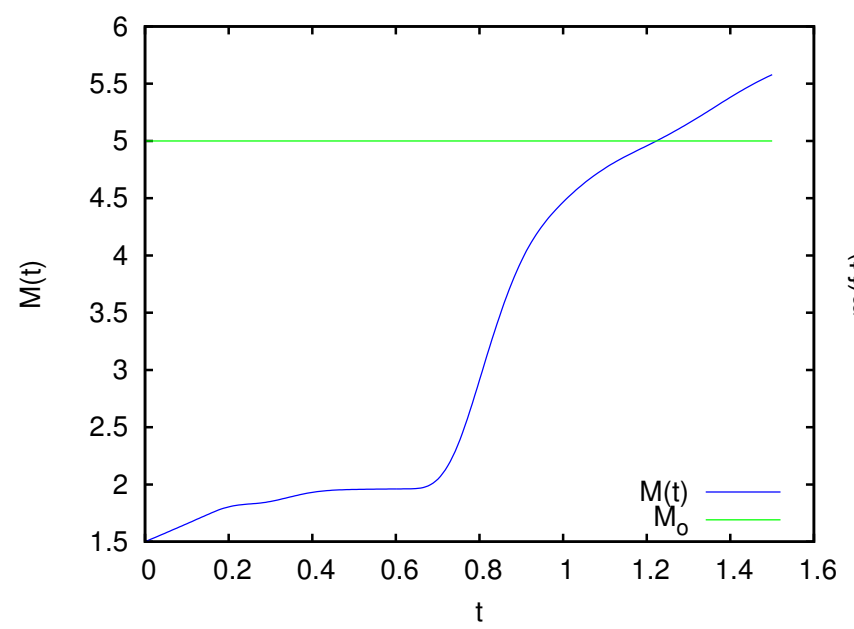

d) Ovarian maturity

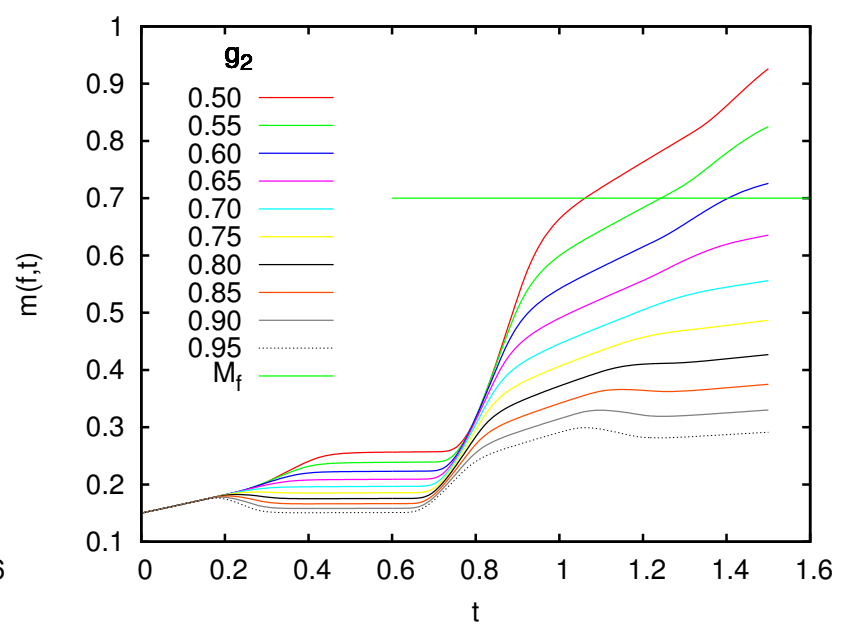

e) Maturity of each follicle

Figure 6 . Competition between ten follicles differing by their ageing function parameter $g_{2}$ 


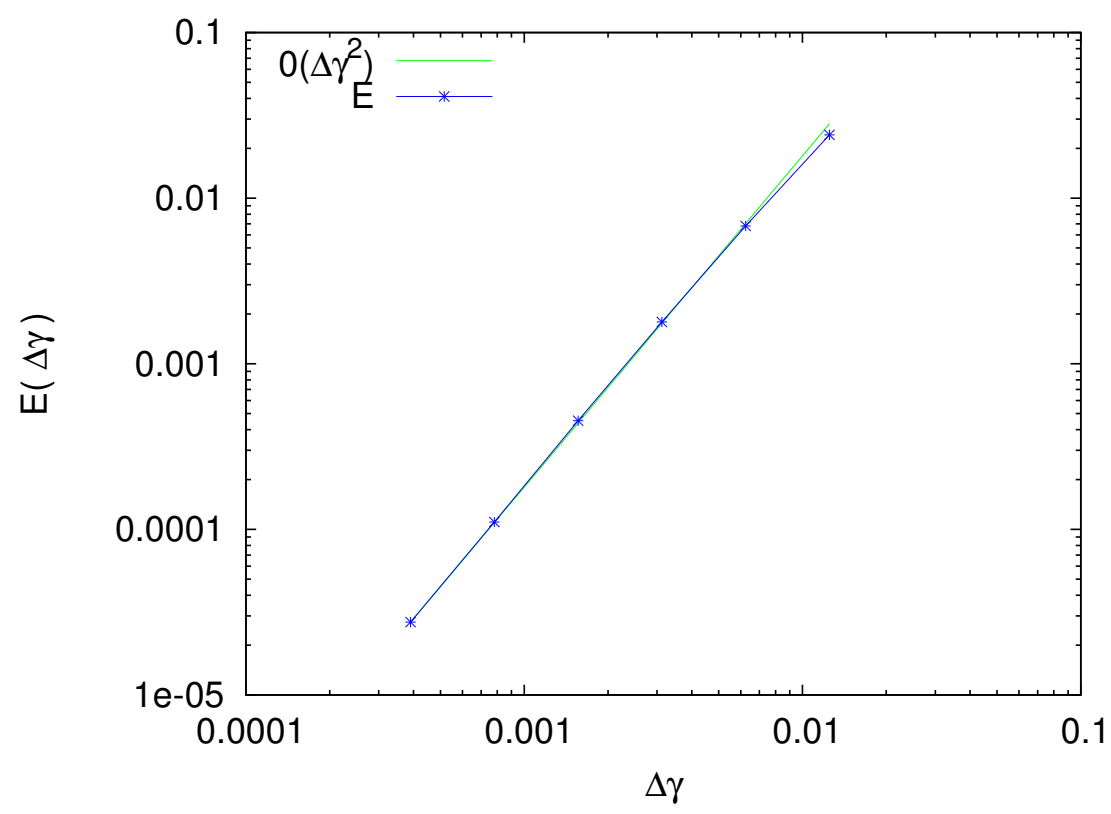

Figure 7. Convergence rate in the SM phase. Green curve : $\Delta \gamma \rightarrow O\left(\Delta \gamma^{2}\right)$. Blue curve : L1 norm error between the exact solution and the solution with space step $\Delta \gamma$.

\subsubsection{Full model convergence test}

We now set the initial condition near the center $C=(0.2,0.15)$ of the $G 1$ phase, still with a maximum time $t_{\max }=0.05$ ensuring that the cell density remains in this zone. In that case the source term (4) and the age and maturity speeds are no more constant, and we no longer know the exact solution of the PDE. The error is computed using the solution with $N_{\gamma}=5120$ as reference solution, that is compared to the solution on the other meshes. Since we have used a dyadic refinement, the size $N_{r e f}$ of the reference mesh in one direction is always a power of two times the size $N_{\Delta \gamma}$ of the current mesh. Denoting by $P=\frac{N_{r e f}}{N_{\Delta \gamma}}$ the ratio between the current discretization and the reference finest one, we estimate the discretization error by

$$
E(\Delta \gamma)=\Delta \gamma^{2} \sum_{k=0}^{N_{\Delta \gamma}-1} \sum_{l=0}^{N_{\Delta \gamma}-1}\left|\phi_{k, l}^{N_{t}}-\frac{1}{P^{2}} \sum_{p=0}^{P} \sum_{m=0}^{P} \phi_{k P+p, l P+m}^{r e f}\right|
$$

The asymptotic order $O\left(\Delta \gamma^{1.95}\right)$ which best fits the behavior of $E(\Delta \gamma)$ is displayed in Figure 8 .

\subsection{HPC test}

The improvement in terms of computing time provided by the parallelization is tested through a set of simulations involving an increasing number of identical follicles. The grid for these simulations consists in one cycle of $200 \times 200$ cells, and the simulation goes on for 900 times steps. In this experiment, we disposed of enough processors to do the computing with one follicle by processor and since the time command is used to monitor the computing time, the output of the program has been commented out. Figure 9 a) shows the real computing time, which is basically the time elapsed from the beginning of the computation. Figure $9 \mathrm{~b}$ ) shows the user computing time, which cumulates all the processors computing time. The computing time cannot be shorter than that required for a computation involving only one, this constitutes the so-called theoretical limit. The fact 


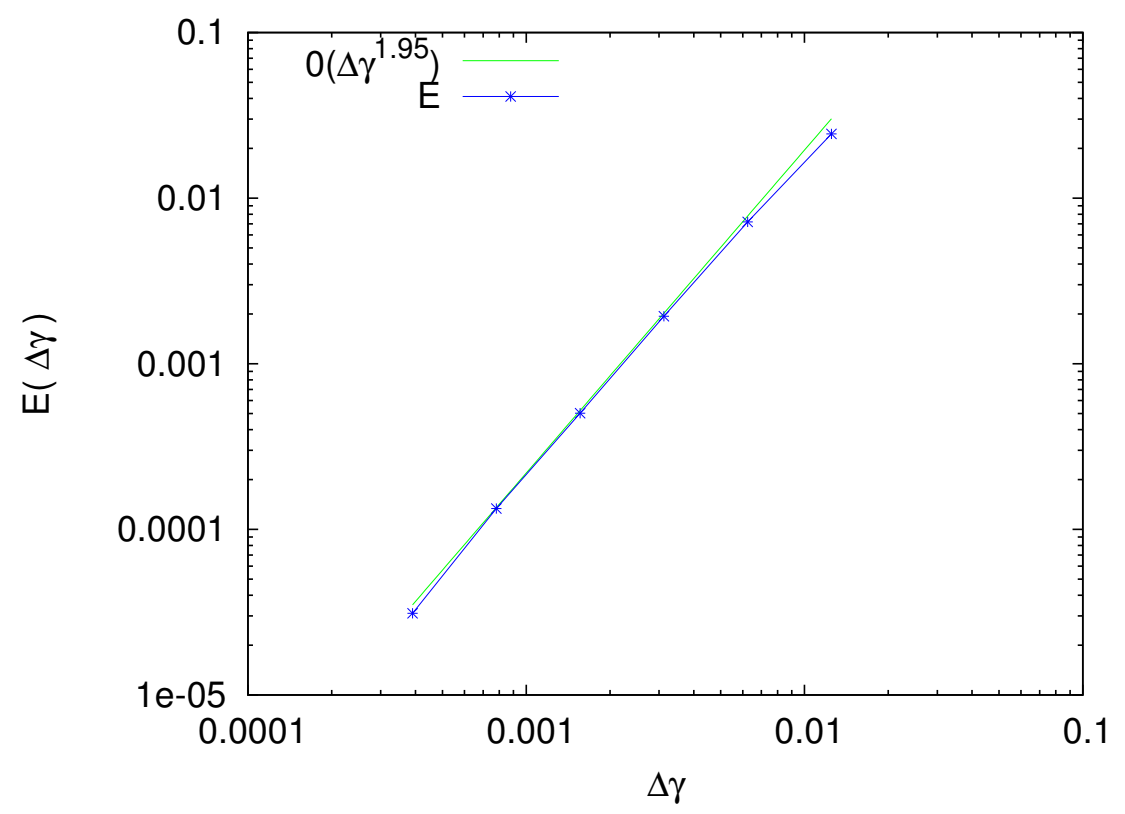

Figure 8. $C V$ rate on the $G 1$ phase. Green curve : $\Delta \gamma \rightarrow O\left(\Delta \gamma^{1.95}\right)$. Blue curve : L1 norm error between the reference solution and the solution with a space step $\Delta \gamma$.

that the real computing time remains close to the computing time for one follicle and that the user computing time grows linearly with the number of follicles is very encouraging. The communications between processors appear not to affect significantly the gain in computing time due to the parallel computing. A small increase in real computing time can be noticed beyond eight follicles. This is due to the super calculator architecture, where the processors are pooled in eight processor nodes. The communications within one node are faster than across different nodes. This leads to a threshold effect observed as soon as more than eight processors are needed, therefore involving more than one single node.

\section{CONCLUSION}

This paper summarizes preliminary works in the development of a dedicated software to illustrate numerically the development of follicles. The uniform grid numerical method have been successfully tested in terms of robustness, accuracy and scalability on parallel architecture. As part of a challenging project involving biologists mathematicians and computer scientists, the different situations engendered by the model (mono-ovulation, poly-ovulation or anovulation) from given combinations of parameters will now be systematically and intensively tested. In order to achieve this goal within realistic delay the HPC aspect of the method must be enriched using adaptive mesh refinement. The multiresolution method developed in [5] and further tested and extended in [6] or [11] is currently implemented in this new configuration, where the presence of source terms and integral terms will require special considerations as exemplified for instance in 2 .

\section{ACKNOWLEDGMENT}

The authors would like to thanks 

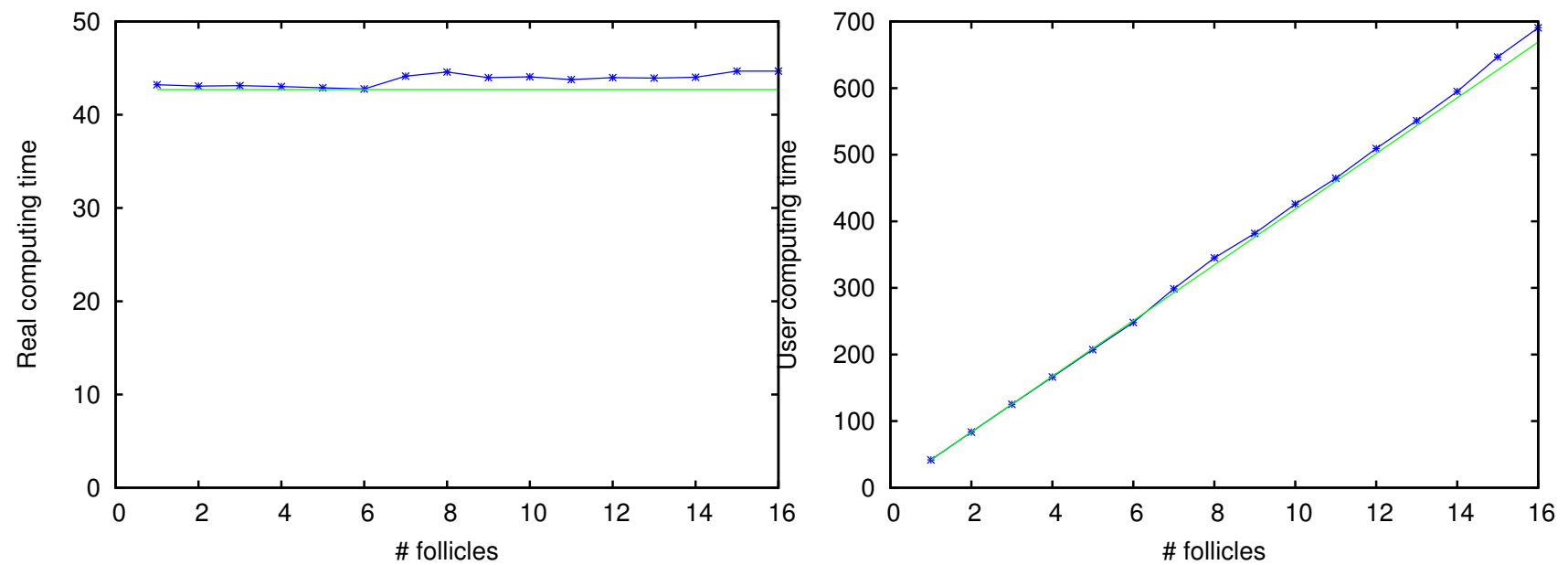

a) Real computing time

c) User computing time

Figure 9. Parallelization performance test. Simulation of an increasing number of identical follicles. One follicle per processor. Green curve : theoretical limit. Blue curve: computing time (in seconds). Left : elapsed time from the start of the computation. Right : cumulative computed time on all processors

- Pascal Joly, Philippe Parnaudeau and Kyrill Pichon Gostaf $f^{3}$ for their help concerning parallel computing,

- the CIRM ${ }^{4}$ for the excellent working conditions,

- the INRIA Large Scale Initiative Action REGATE5 ${ }^{5}$ for the funding of the project.

\section{REFERENCES}

[1] F. Bouchut and F. James. One-dimensional transport equations with discontinuous coefficients. Nonlinear Anal., 32(7):891-933, 1998.

[2] G. Chiavassa, R. Donat, and A. Martinez-Gavara. Cost-effective multiresolutions schemes for shock computations. In Multiresolution and adaptive methods for convection-dominated problems, volume 29 of ESAIM Proc., pages 8-27. EDP Sci., Les Ulis, 2009.

[3] F. Clément. Multiscale modelling of endocrine systems: new insight on the gonadotrope axis. ESAIM: Proc., 27:209-226, 2009.

[4] F. Clément, J.-M. Coron, and P. Shang. Optimal control for multiscale conservation laws describing the development of ovarian follicles. 2011.

[5] A. Cohen, S. M. Kaber, S. Müller, and M. Postel. Fully adaptive multiresolution finite volume schemes for conservation laws. Math. Comp., 72(241):183-225 (electronic), 2003.

[6] F. Coquel, Q. L. Nguyen, M. Postel, and Q. H. Tran. Entropy-satisfying relaxation method with large time-steps for Euler IBVPs. Math. Comp., 79(271):1493-1533, 2010.

[7] N. Echenim. Modélisation et contrôle multi-échelles du processus de sélection des follicules ovulatoires. PhD thesis, Université Paris-Sud XI, Faculté des Sciences d'Orsay, 2006.

[8] N. Echenim, F. Clément, and M. Sorine. Multiscale modeling of follicular ovulation as a reachability problem. Multiscale Model. Simul., 6(3):895-912, 2007.

[9] N. Echenim, D. Monniaux, M. Sorine, and F. Clément. Multi-scale modeling of the follicle selection process in the ovary. Math. Biosci., 198(1):57-79, 2005.

[10] E. Godlewski and P.-A. Raviart. The numerical interface coupling of nonlinear hyperbolic systems of conservation laws. I. The scalar case. Numer. Math., 97(1):81-130, 2004.

\footnotetext{
${ }^{3}$ Laboratoire Jacques-Louis Lions, UPMC-Paris 6

${ }^{4}$ http://www.cirm.univ-mrs.fr

${ }^{5}$ https://www.rocq.inria.fr/sisyphe/reglo/regate.html
} 
[11] N. Hovhannisyan and S. Müller. On the stability of fully adaptive multiscale schemes for conservation laws using approximate flux and source reconstruction strategies. IMA J. Numer. Anal., 30(4):1256-1295, 2010.

[12] R.J. LeVeque. Wave propagation algorithms for multidimensional hyperbolic systems. J. Comput. Phys., 131(2):327-353, 1997.

[13] R.J. LeVeque and M.J. Berger. Adaptive mesh refinement using wave-propagation algorithms for hyperbolic systems. SIAM J. Numer. Anal., 35(6):2298-2316, 1998.

[14] P. Michel. Multiscale modeling of follicular ovulation as a mass and maturity dynamical system. Multiscale Model. Simul., 9(1):282-313, 2011.

[15] P. Shang. Cauchy problem for multiscale conservation laws : Applications to structured cell populations. http://arxiv.org/abs/1010.2132, 2010.

[16] P. K. Sweby. High resolution schemes using flux limiters for hyperbolic conservation laws. SIAM J. Numer. Anal., 21(5):9951011, 1984 